\title{
Advancing cancer treatment: A move towards individualized therapy
}

\section{Taylor F. Bureyko and Rachel A. Murphy}

\author{
University of Alberta
}

In Canada, cancer has surpassed heart disease as the leading cause of death. ${ }^{1}$ The incidence of cancer and cancer-related deaths is increasing. ${ }^{1}$ This is likely to continue to increase in the coming years given our aging population and the fact that cancer primarily affects people over the age of $50 .{ }^{1}$ Each year, the government and voluntary sectors spend over $\$ 400$ million on cancer research in Canada. ${ }^{2}$ Although progress has been made in the treatment of certain types of cancer, we are still far from being able to offer all patients effective treatment.

The primary goal of an oncologist is to recommend the most effective cancer treatment available. However, it is difficult to predict patient response or resistance to therapeutic agents. In order to maximize benefits from treatment, specifically to improve quality of life and to prolong survival, we must understand and address variability in treatment response. Molecular differences in malignant tissue may explain some of the heterogeneity in treatment response and provide novel treatment targets.

Currently, patients receive standardized anti-neoplastic therapy according to tumour histology and disease stage. Advancements in molecular profiling and drug development have led to the possibility of individualizing treatment according to the molecular characteristics of a patient's tumour. These new therapies target specific cellular features that are essential for tumour growth or survival. Due to the specific nature of these therapies, the side effects from targeted therapy are often milder than conventional antineoplastic treatments. ${ }^{3}$ Together, molecular profiling and targeted therapy may improve upon standardized treatment by identifying molecular characteristics associated with response or resistance to therapeutic agents.
Targeted therapy in lung cancer is an area of intense research due to low efficacy of standard chemotherapy. The drugs erlotinib and gefitinib, which inhibit tyrosine kinase activity in the epidermal growth factor receptor (EGFR), are examples of targeted therapy. Response to these therapies has been associated with mutations in the tyrosine kinase region of EGFR which are particularly prevalent in Asian women with no smoking history who develop adenocarcinoma of the lung. ${ }^{4,5}$ Thus, erlotinib and gefitinib are most effective in this particular population. ${ }^{6}$ Molecular profiling may also be useful for selecting the most effective treatment for patients without EGFR mutations as these patients have been shown to benefit from standard chemotherapy compared to gefitinib.

Similarly, targeted therapies have been successful in improving treatment for breast cancer. Approximately $20 \%$ of patients with breast cancer overexpress a growth factor receptor gene, human epidermal growth factor receptor (HER2), which is associated with aggressive disease and higher risk of cancer recurrence. ${ }^{8}$ The development of trastuzumab, a monocolonal antibody which interferes with the HER2 receptor, has resulted in longer progression-free survival and significant improvements in survival in HER2-positive breast cancer patients. ${ }^{9}$ This represents a major advancement in treatment of breast cancer and has contributed to a $25 \%$ decline in mortality from breast cancer in Canadian women over the last two decades. ${ }^{1}$

Although targeted therapy seems promising, there are concerns about the feasibility of an individualized approach to cancer treatment. These concerns are centered on obtaining and characterizing tumour biopsies in a timely manner. However, these concerns may be unfounded as a recent study in advanced cancer patients obtained tumour biopsies for all study patients $(n=86)$ 
from 9 different cancer centers. ${ }^{10}$ Molecular profiling was then used to identify treatment targets and to select treatment regimens. All patients had refractory disease, having previously failed to respond to chemotherapy. Despite this, $27 \%$ of patients had longer progression-free survival with individualized treatment compared to their previous treatment regimens. Marked differences between therapies that would have been recommended by the patients' oncologist in the absence of molecular profiling were also reported. This study not only demonstrates that individualized cancer therapy is feasible but that it may also represent an improvement over standard treatment.

Current knowledge of the complex interactions between specific gene expression and targeted treatment is evolving, as is molecular profiling technology. Great advancements in treatment have already been made with the advent of targeted molecular agents such as trastuzumab, gefitinib and erlotinib. These agents have fewer side effects and provide more effective disease control than standardized therapy in subgroups of patients. Although thus far, molecular agents are most effective in well-defined subsets of patients, further development of targeted therapies will open new avenues in treatment for broader populations. Continued research and development of novel molecular targets and treatments are needed, but the encouraging results to date suggest that individualized anti-neoplastic therapy holds promise for advancing the treatment of cancer.

\section{References}

1. Canadian Cancer Society's Steering Committee: Canadian Cancer Statistics 2010. Toronto: Canadian Cancer Society, 2010.

2. Canadian Cancer Research Alliance (2008). Cancer Research Investment in Canada, 2006: The Canadian Cancer Research Alliance's survey of government and voluntary sector investment in cancer research in 2006.Toronto: CCRA.

3. Shepherd FA, Pereira JR, Ciuleanu T, et al. Erlotinib in previously treated non-small cell lung cancer. N Engl J Med 2005; 353:123-132.

4. Kosaka T, Yatabe Y, Endoh H, Kuwano H, Takahashi T, Mitsudomi T. Mutations of the epidermal growth factor receptor gene in lung cancer: biological and clinical implications. Cancer Res 2004; 64:8919-23.

5. Shigematsu H, Lin L, Takahashi T, et al. Clinical and biological features associated with epidermal growth factor receptor gene mutations in lung cancer. J Natl Cancer Inst 2005; 97:39-46.

6. Shepherd FA, Rodrigues Pereira J, Ciuleanu T, et al. Erlotonib in previously treated non-small-cell lung cancer. N Engl J Med 2005; 353:123-32.
7. Mok TS, Wu YL, Thongprasert S, et al. Gefitinib or carboplatin-paclitaxel in pulmonary adenocarcinoma. N Engl J Med 2009; 361:947-57.

8. Slamon DJ, Clark GM, Wong SG, Levin WJ, Ullrich A, McGuire WL. Human breast cancer: correlation of relapse and survival with amplification of the HER22/neu oncogene. Science 1987; 235:177-182.

9. Slamon DJ, Leyland-Jones B, Shak S, et al. Use of chemotherapy plus a monoclonal antibody against HER2 or metastatic breast cancer that overexpresses HER2. N Engl J Med 2001; 344(11):783-92.

10. von Hoff DD, Stephenson JJ Jr, Rosen P, et al. Pilot study using molecular profiling of patients' tumors to find potential targets and select treatments for their refractory cancers. J Clin Oncol 2010; 28:4877-83.

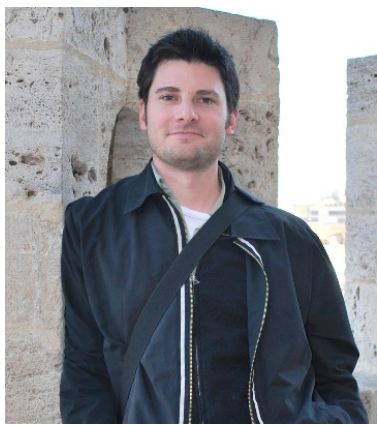

\section{Author Profiles}

Taylor Bureyko is currently a $\mathrm{PhD}$ candidate in the Department of Agricultural, Food and Nutritional Science at the University of Alberta. His research interests include n-3 polyunsaturated fatty acids, integrins and prostate cancer.

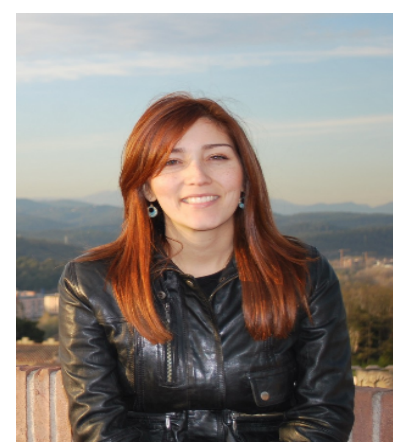

Rachel Murphy is currently pursuing a $\mathrm{PhD}$ in Nutrition and Metabolism from the University of Alberta. Her research interests are in nutrition and cancer, supportive care, geriatrics and muscle and adipose tissue metabolism in aging and chronic disease. Rachel received her BSc in Biology from the University of Guelph and will be starting a fellowship at the National Institute of Health this fall. 